\title{
How to best fold a triangle
}

\author{
Christer O. Kiselman
}

Received: 28 December 2016 / Accepted: 3 March 2017 / Published online: 12 May 2017

(C) The Author(s) 2017. This article is an open access publication.

\begin{abstract}
We fold a triangle once along a straight line and study how small the area of the folded figure can be. It can always be as small as the fraction $2-\sqrt{2}$ of the area of the original triangle.
\end{abstract}

This is best possible: For every positive number $\varepsilon$ there are triangles that cannot be folded better than $2-\sqrt{2}-\varepsilon$.

\section{The question}

Suppose we have a paper in the form of a triangle. We fold it once along a straight line and ask how small the area of the folded object can be - equivalently, how large the area of the doubly covered part can be. Clearly this latter area cannot be larger than one half of that of the triangle, and it is exactly one half if and only if the triangle is isosceles. But how large can the area of the doubly covered part be if the triangle is scalene? And how small is the area in the worst possible case?

We may obviously ask what happens if we fold polygons with four, five, six, ... sides. As Bo Senje reports, problems on the folding of rectangles have been given

C. O. Kiselman $(\bowtie)$

Department of Information Technology, Uppsala University, P.O. Box 337, 75105 Uppsala,

Schweden

E-Mail: kiselman@it.uu.se 
to students. But rectangles are quite special quadrilaterals. And then we should pass to polyhedra in $n \geq 3$ dimensions ...

\section{The answer}

Theorem 1 Every triangle can be folded along a straight line so that the area of the doubly covered part is the fraction $\sqrt{2}-1 \approx 0.4142$ of the area of the original triangle. This is best possible in the sense that, given any positive number $\varepsilon$, there exist triangles that cannot be folded to yield an area of the doubly covered part larger than the fraction $\sqrt{2}-1+\varepsilon$.

The area of the folded figure and the area of its doubly covered part sum up to the area of the original triangle. To ask for the smallest area of the folded figure is therefore equivalent to asking for the largest area of the doubly covered part. For the calculations to be made, it is easier to consider the latter quantity, which we shall do from now on. (See, e. g., Proposition 5. In the simple but important estimate in Proposition 7, the folded object has infinite area, so we can only work with the area of the doubly covered part of the strip.)

Denote the side lengths of the triangle by $A, B$ and $C$, with the notation chosen so that $A \leq B \leq C$.

We shall fold a triangle in two different ways:

1. If we fold along the bisector of the smallest angle, then the relative area of the doubly covered part is $B /(B+C)$. This quantity is $\geq \sqrt{2}-1$ if and only if

$$
B \geq C / \sqrt{2} \text {. }
$$

2. If we fold along a line which is orthogonal to the longest side, then the best result to be obtained is $C^{2} /\left(B^{2}-A^{2}+2 C^{2}\right)$. This quantity is $\geq \sqrt{2}-1$ if and only if

$$
B^{2} \leq A^{2}+(\sqrt{2}-1) C^{2}
$$

One of (1) and (2) always holds.

So to obtain the fraction $\sqrt{2}-1$, we need two different kinds of folding: along lines that pass through a vertex and lines that are orthogonal to a side. Since we use only special foldings for these considerations, calculations are not very complicated.

To prove that it is not possible to do anything better, we must consider all foldings, so calculations become more involved. We shall study triangles with vertices at $a=(0,0), b=(1,0)$, and $c=\left(c_{1}, c_{2}\right)$, with $c_{1}=\frac{1}{2} \sqrt{2}$ and $c_{2}>0$ very small. This means that the side lengths $A: B: C$ are almost proportional to $\left(1-\frac{1}{2} \sqrt{2}\right): \frac{1}{2} \sqrt{2}: 1$. These narrow triangles will give us the examples where all foldings are bad.

When the line avoids the direction of the longest side as well as a direction orthogonal to the longest side, a simple estimate shows that the area of the doubly covered part cannot be close to $\sqrt{2}-1$ (Sect. 9.1 and Fig. 2). The situations when the 
line is almost parallel or almost orthogonal to the longest side of these thin triangles remain to be studied. This is done in Sects. 9.2 and 9.3, respectively.

\section{The history}

Sometime during the years 1997-1999, Bo Senje at Halmstad University College asked a question: How large can the doubly covered part of a folded triangle be? He had seen some student problems on the folding of rectangles but, to his surprise, never any on the folding of triangles. (Now we may suspect that the explanation was that triangles are more difficult than rectangles.)

Georgi Mihailov Tchilikov (1967-2010), also at Halmstad University College, worked on the problem from time to time since December 2004. Some time later (according to Bo most probably in 2008) he found formula (19) for foldings along a bisector.

Bo also asked: Will the best folding always go through a vertex of the triangle?

The answer to this second question is in the negative as we shall see: for certain triangles, folding orthogonally to a side gives better results than folding along a bisector (see Remark 1). Also Georgi had found this. His results have not been published.

The questions posed by Bo reached me in April 2005. I did not know about the results proved by Georgi until February 2015.

Independently of what was known in Halmstad, I proved (in 2005 or 2006) the formula for foldings along lines through a vertex, and Martin Herschend then found a triangle where folding orthogonally to a side gives a better result than folding along a line through a vertex.

After having tried many different ways to approach the question - too many to be remembered - I could finally prove in February 2015 that $\sqrt{2}-1$ is best possible. The proof was, however, so cumbersome that I did not dare to submit it to any journal. Only in the evening of 2016 October 13 did I find a proof that might convince more than one person. Of course I cannot exclude that there exist simpler proofs.

I thank Bo Senje for having asked these questions - a most important activity and the starting point of any kind of research. He also checked an earlier version of the manuscript and sent me comments.

I am grateful to two anonymous referees, whose comments have led to clarifications in the presentation.

For origamics in general, see [1] and [3]. More recently, [4, 5], and [2] studied paper foldings. The first-mentioned article treats mostly folding of rectangles, then of right-angled triangles, and finally of general triangles. However, the best possible result is not obtained. Oswald's paper [5] is about results on paper foldings obtained by Adolf Hurwitz (1859-1919). 


\section{Calculating with triangles}

\subsection{Best foldings and the worst best folding}

Let $T$ be a triangle, understood as the convex hull of three non-collinear points in the plane, and let $\operatorname{area}(T)$ be its area. We shall denote by $T(a, b, c)$ the triangle with vertices $a, b, c$.

We denote by $Y(\varphi, t)$ the half plane defined by

$$
-x_{1} \sin \varphi+x_{2} \cos \varphi \leq t, \quad \varphi \in \mathbf{R}, t \in \mathbf{R},
$$

and by $L(\varphi, t)$ its boundary, defined by

$$
-x_{1} \sin \varphi+x_{2} \cos \varphi=t, \quad \varphi \in \mathbf{R}, t \in \mathbf{R},
$$

forming an angle $\varphi$ with the positive $x_{1}$-axis. Obviously $Y(\varphi+2 \pi, t)=Y(\varphi, t)$ and $L(\varphi+\pi, t)=L(\varphi,-t)$.

Let $Y$ be a half plane and let $T^{[\partial Y]}=T^{[L]}$ be the reflection of $T$ in its boundary $\partial Y=L$. We fold $T$ along $L$ and place the folded object in $Y$. Then the part which is doubly covered is $T \cap T^{[\partial Y]} \cap Y$. We denote it by $T_{Y}$. It is in general a quadrilateral. The folded object is $\left(T \cup T^{[\partial Y]}\right) \cap Y$, to be denoted by $T^{Y}$. It is in general a heptagon. See Figs. 1 and 4, respectively.

Depending of the shape of the triangle and the position of the line, some geometric elements can coincide: the doubly covered part can be a triangle; the folded object can have less than seven sides: six, five, four, or even three sides. See Fig. 3 for an example where $T_{Y}$ is a quadrilateral and $T^{Y}$ has five vertices. These special cases need not be treated separately since the calculations in the general case cover them all.

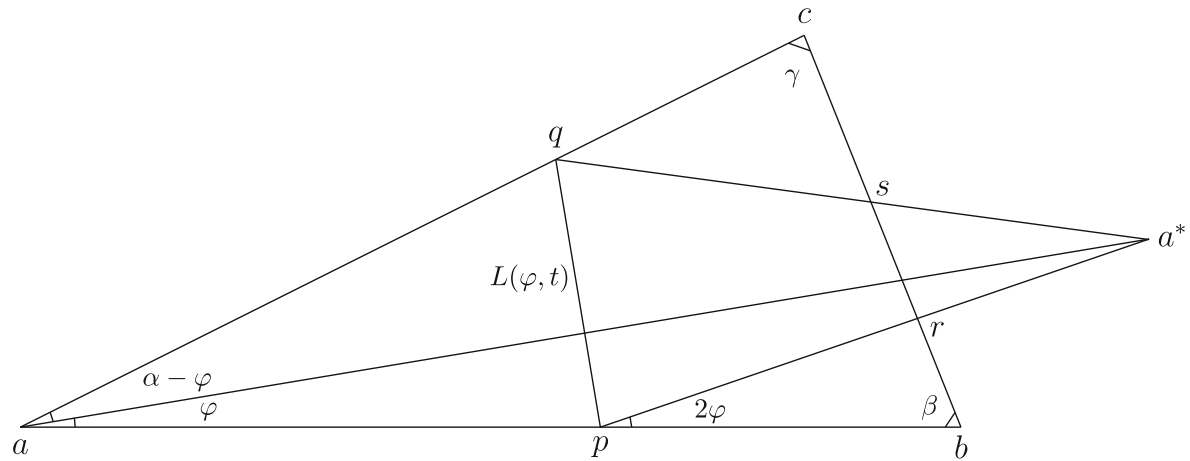

Fig. 1 The original triangle $T(a, b, c)$ has its vertices at $a, b$ and $c$. We fold along a straight line $L(\varphi, t)$, which intersects $[a, b]$ in a point $p$ and $[c, a]$ in a point $q$, and place the folded object to the right of the line. The doubly covered set $T_{Y}$ is then a quadrilateral $Q(p, r, s, q)$ with vertices at $p, r, s$, $q$. The folded object $T^{Y}$ is a heptagon with vertices at $p, b, r, a^{*}, s, c, q$, where $a^{*}$ is the reflection of $a$ in the line $L(\varphi, t)$. In the figure, the parameters $\varphi$ and $t$ defining the line $L(\varphi, t)$ are chosen to satisfy $0<\varphi<\alpha<\frac{1}{2} \pi$ and $a_{1}<p_{1}=-t / \sin \varphi<b_{1}$ 
We have

$$
\operatorname{area}\left(T_{Y}\right)+\operatorname{area}\left(T^{Y}\right)=\operatorname{area}(T \cap Y)+\operatorname{area}\left(T^{[\partial Y]} \cap Y\right)=\operatorname{area}(T),
$$

so that the relative areas satisfy

$$
\frac{\operatorname{area}\left(T_{Y}\right)}{\operatorname{area}(T)}+\frac{\operatorname{area}\left(T^{Y}\right)}{\operatorname{area}(T)}=1
$$

The problem is to maximize the area of the set $T_{Y}$ which is doubly covered.

We define the best folding of $T$ as the largest relative area of the doubly covered object,

$$
\mathrm{BF}(T)=\sup _{Y} \frac{\operatorname{area}\left(T_{Y}\right)}{\operatorname{area}(T)},
$$

where the supremum is taken over all possible half planes $Y$. For each triangle, the supremum is attained for at least one choice of $Y$. The quantity $\operatorname{BF}(T)$ satisfies

$$
0<\mathrm{BF}(T) \leq \frac{1}{2}
$$

As already noticed, the second inequality in (6) is an equality if and only if $T$ is isoceles and is realized when $L=\partial Y$ is the bisector between two equal sides.

How bad can $\operatorname{BF}(T)$ be? Let us define the worst best folding WBF as

$$
\mathrm{WBF}=\inf _{T} \mathrm{BF}(T)=\operatorname{infsup}_{T} \frac{\operatorname{area}\left(T_{Y}\right)}{\operatorname{area}(T)} .
$$

\subsection{Standard coordinates}

It will often be convenient use the following coordinate system.

A triangle in standard coordinates:

The vertices are $a=(0,0), b=(1,0)$ and $c=\left(c_{1}, c_{2}\right) \in \Gamma$, where

$$
\Gamma=\left\{c \in \mathbf{R}^{2} ; \quad \frac{1}{2} \leq c_{1}<1, c_{2}>0, c_{1}^{2}+c_{2}^{2} \leq 1\right\} .
$$

The angles at the vertices $a, b, c$ are denoted by $\alpha \leq \beta \leq \gamma$.

Thus all possible triangles are represented by the point $c$ in the set $\Gamma$, which is bounded by the segments $\left[\left(\frac{1}{2}, \frac{1}{2} \sqrt{3}\right),\left(\frac{1}{2}, 0\right)\right]$ and $\left[\left(\frac{1}{2}, 0\right),(1,0)\right]$, and the circular arc from $(1,0)$ to $\left(\frac{1}{2}, \frac{1}{2} \sqrt{3}\right)$ with center at the origin and of radius 1 . The triangles with $c_{1}=\frac{1}{2}$ as well as those with $c$ on the circular arc are isoceles, and for them $\mathrm{BF}(T)=\frac{1}{2}$. 
We shall prove that, in standard coordinates,

$$
\mathrm{BF}(T) \geq \max \left(\frac{1}{1+1 /\|c\|_{2}}, \frac{1}{2 c_{1}+1}\right), \quad c \in \Gamma .
$$

The right-hand side is larger than $\sqrt{2}-1$ for all $c \in \Gamma$. It follows that $\mathrm{BF}(T)>\sqrt{2}-1$ for all triangles $T$, and that $\mathrm{WBF} \geq \sqrt{2}-1$. Thus the first part of the main theorem follows.

The second part of the main theorem, to be proved in Sect. 9, says that the infimum of $\mathrm{BF}(T)$ over all triangles $T$ is equal to $\sqrt{2}-1$. It follows that $\mathrm{WBF}=\sqrt{2}-1$.

The right-hand side in (9) can be extended to a continuous function of $c \in \bar{\Gamma}=$ $\Gamma \cup[(1 / 2,0),(1,0)]$, and this extension takes the value $\sqrt{2}-1$ only at $c=(1 / \sqrt{2}, 0)$. Hence the bad triangles are only those with $c$ close to $(1 / \sqrt{2}, 0)$. So, the infimum defining WBF is not attained: there is no worst triangle, but a family $\left(T_{\varepsilon}\right)_{\varepsilon>0}$ of bad triangles such that $\mathrm{BF}\left(T_{\varepsilon}\right)$ tends to $\mathrm{WBF}$ as $\varepsilon \searrow 0$; in standard coordinates, the vertex $c \in \Gamma$ must tend to $(1 / \sqrt{2}, 0) \notin \Gamma$.

\subsection{A calculus of points and lines}

As already mentioned, the vertices of a triangle will be called $a, b, c$. Its angles will be denoted by $\alpha, \beta, \gamma$; its side lengths $A=\|b-c\|_{2}, B=\|c-a\|_{2}, C=\|a-b\|_{2}$, where $\|x\|_{2}=\sqrt{x_{1}^{2}+x_{2}^{2}}$ denotes the Euclidean norm of a vector $x$. Often, but not always, we shall choose notation so that $A \leq B \leq C$. Then also $\alpha \leq \beta \leq \gamma$, and $\sin \alpha \leq \sin \beta \leq \sin \gamma=\sin (\alpha+\beta)$. We have

$$
0<\alpha \leq \pi / 3,0<\beta<\pi / 2, \pi / 3 \leq \gamma<\pi
$$

In addition to these inequalities we have $\alpha \leq \beta \leq \frac{1}{2} \pi-\frac{1}{2} \alpha$, so that the admissible pairs $(\alpha, \beta)$ belong to the closed triangle with vertices at $(0,0),\left(\frac{1}{3} \pi, \frac{1}{3} \pi\right)$ and $\left(0, \frac{1}{2} \pi\right)$ with the segment $\left[(0,0),\left(0, \frac{1}{2} \pi\right)\right]$ removed.

We shall need some explicit calculations on lines and reflections in lines. We study half planes $Y(\varphi, t)$ defined by (3) and lines $L(\varphi, t)$ defined by (4).

Two straight lines $L\left(\varphi_{1}, t_{1}\right)$ and $L\left(\varphi_{2}, t_{2}\right)$ with $\varphi_{1}-\varphi_{2} \notin \pi \mathbf{Z}$ meet in a unique point

$$
y=\left(\frac{t_{2} \cos \varphi_{1}-t_{1} \cos \varphi_{2}}{\sin \left(\varphi_{1}-\varphi_{2}\right)}, \frac{t_{2} \sin \varphi_{1}-t_{1} \sin \varphi_{2}}{\sin \left(\varphi_{1}-\varphi_{2}\right)}\right) .
$$

Two different points $p$ and $q$ determine a line $L(\theta, s)$ with

$$
\theta=\arccos \left(\frac{p_{1}-q_{1}}{\|p-q\|_{2}}\right)=\arcsin \left(\frac{p_{2}-q_{2}}{\|p-q\|_{2}}\right) \text { and } s=\frac{p_{1} q_{2}-p_{2} q_{1}}{\|p-q\|_{2}} .
$$


By reflection in the straight line $L(\varphi, t)$, a point $p$ is mapped to a point $p^{*}$, which is given by

$$
\left(\begin{array}{l}
p_{1}^{*} \\
p_{2}^{*}
\end{array}\right)=\left(\begin{array}{cc}
\cos 2 \varphi & \sin 2 \varphi \\
\sin 2 \varphi & -\cos 2 \varphi
\end{array}\right)\left(\begin{array}{l}
p_{1} \\
p_{2}
\end{array}\right)+2 t\left(\begin{array}{c}
-\sin \varphi \\
\cos \varphi
\end{array}\right) .
$$

A line $L\left(\theta_{1}, s_{1}\right)$, when reflected in a line $L(\varphi, t)$, is mapped to the line $L\left(\theta_{2}, s_{2}\right)$, where

$$
\theta_{2}=2 \varphi-\theta_{1} \text {, and } s_{2}=2 t \cos \left(\varphi-\theta_{1}\right)-s_{1} .
$$

\subsection{A calculus of areas}

We shall use the notation $\rho_{T}(\varphi, t)$ for the relative area of $T_{Y(\varphi, t)}$, thus

$$
\rho_{T}(\varphi, t)=\frac{\operatorname{area}\left(T_{Y(\varphi, t)}\right)}{\operatorname{area}(T)}, \quad(\varphi, t) \in \mathbf{R} \times \mathbf{R} .
$$

We can take the supremum in two steps:

$$
\mathrm{BF}(T)=\sup _{\varphi \in \mathbf{R}} \sup _{t \in \mathbf{R}} \rho_{T}(\varphi, t)=\sup _{\varphi \in \mathbf{R}} \sigma_{T}(\varphi),
$$

where

$$
\sigma_{T}(\varphi)=\sigma_{T}(\varphi+\pi)=\sup _{t \in \mathbf{R}} \rho_{T}(\varphi, t), \quad \varphi \in \mathbf{R},
$$

the best that can be obtained if we fold only along lines parallel to a fixed line.

If the vertices $a, b, c$ are taken in the positive direction, the triangle $T(a, b, c)$ has the area

$$
\operatorname{area}(T(a, b, c))=\frac{1}{2}(|a b|+|b c|+|c a|),
$$

where we think of $a \in \mathbf{R}^{2}$ as a column vector, $a=\left(a_{1}, a_{2}\right)^{\mathrm{T}}$, and write

$$
|a b|=\operatorname{det}\left(\begin{array}{ll}
a_{1} & b_{1} \\
a_{2} & b_{2}
\end{array}\right)=a_{1} b_{2}-a_{2} b_{1} .
$$

A quadrilateral with vertices $a, b, c, d$ will be denoted by $Q(a, b, c, d)$. If the vertices are taken in the positive direction, it has the area

$$
\operatorname{area}(Q(a, b, c, d))=\frac{1}{2}(|a b|+|b c|+|c d|+|d a|) .
$$




\subsection{Special foldings}

We shall also briefly discuss quantities $\mathrm{BF}^{\text {vertex }}(T), \mathrm{BF}^{\perp}(T), \mathrm{WBF}^{\text {vertex }}$, and $\mathrm{WBF}^{\perp}$, defined in analogy with $\mathrm{BF}(T)$ and $\mathrm{WBF}$, but when we fold only along lines that pass through a vertex or are orthogonal to a side, respectively. Clearly

$$
\mathrm{BF}^{\text {vertex }}(T), \mathrm{BF}^{\perp}(T) \leq \mathrm{BF}(T) \text { for every triangle } T,
$$

yielding $\mathrm{WBF}^{\mathrm{vertex}}, \mathrm{WBF}^{\perp} \leq \mathrm{WBF}$. Actually we have strict inequality here:

$$
\mathrm{WBF}^{\perp}=\frac{1}{3}<\mathrm{WBF}^{\text {vertex }}=\frac{3}{2}-\frac{1}{2} \sqrt{5}<\mathrm{WBF}=\sqrt{2}-1 .
$$

We shall find families $\left(T_{\varepsilon}^{\text {vertex }}\right)_{\varepsilon>0}$ and $\left(T_{\varepsilon}^{\perp}\right)_{\varepsilon>0}$ of bad triangles for these two special foldings.

In the next sections, we shall start by studying some special foldings. We first fold along a straight line which is parallel to a side; see Sect. 5. This kind of folding can never give a better result than $\frac{1}{3}<\sqrt{2}-1$.

To prove that a triangle can always be folded to yield the relative area $\sqrt{2}-1$ of the doubly convered set we shall study two special ways of folding a triangle.

We fold along a line which passes through a vertex; see Sect. 6 and Corollary 2. In this case

$$
\mathrm{BF}^{\text {vertex }}(T)=\max \left(\frac{1}{1+\sin \gamma / \sin \beta}, \frac{1}{1+\sin \beta / \sin \alpha}\right),
$$

where $\alpha \leq \beta \leq \gamma$ are the angles of $T$.

If we fold along a line which is parallel to a bisector, the result is the same, i. e., the supremum of area $\left(T_{Y}\right)$ over all half planes with $L=\partial Y$ parallel to a certain bisector is the same as the supremum of the area over all half planes $Y$ with $L=\partial Y$ passing through the vertex of that bisector.

The bad triangles for this kind of folding are those with vertices in $(0,0),(0,1)$, $\left(c_{1}, c_{2}\right)$, where $c_{1}=\mathbf{A u}$ and $c_{2}$ is a small positive number. Here $\mathbf{A u}$ is the Golden Ratio, equal to $\frac{1}{2}(\sqrt{5}-1) \approx 0.6180$ (also known as the Golden Section or Golden Proportion). For them the best folding tends to $1 /(1+1 / \mathbf{A u})=\frac{3}{2}-\frac{1}{2} \sqrt{5} \approx 0.3820$ (see Corollary 2).

Then we fold along a straight line which is orthogonal to a side (see Sect. 7). It turns out that the worst best folding $\mathrm{WBF}^{\perp}$ for these foldings is $\frac{1}{3}$.

It can happen that the supremum when we keep $L$ parallel to a given direction which is not a bisector is larger than when $L$ passes through a vertex (see Remark 1 ).

Combining two of the methods, viz. folding either along lines through a vertex or orthogonally to a side (a choice depending on the triangle), we find that the universal constant WBF is at least equal to $\sqrt{2}-1 \approx 0.4142$; see (9). 


\section{Folding along a line parallel to a side}

When folding along a line parallel to a side, it matters whether the angles adjacent to the side are at most $\frac{1}{2} \pi$ or whether one is larger then $\frac{1}{2} \pi$. In the first case, the best result is always $1 / 3$, thus smaller than $\sqrt{2}-1$; in the second case it is even smaller than $1 / 3$. Thus folding along a line parallel to a side never yields a good result.

Proposition 2 Let $T$ be the triangle with vertices at $a=(0,0), b=(1,0), c=$ $\left(c_{1}, c_{2}\right)$, assuming $0 \leq c_{1} \leq 1$ and $c_{2}>0$. We fold it along the line $L(0, t)=$ $\partial Y(0, t)$, thus of equation $x_{2}=t$, and put the folded object in the half plane $Y(0, t)$, defined by $x_{2} \leq t$. Then the relative area of

$$
T_{Y(0, t)}=T \cap T^{[\partial Y(0, t)]} \cap Y(0, t)
$$

is

$$
\rho_{T}(0, t)=\frac{\operatorname{area}\left(T_{Y(0, t)}\right)}{\operatorname{area}(T)}= \begin{cases}0 & t \leq 0 \\ \frac{1}{3}-\frac{3}{c_{2}^{2}}\left(t-\frac{1}{3} c_{2}\right)^{2}, & 0 \leq t \leq \frac{1}{2} c_{2} \\ \frac{1}{c_{2}^{2}}\left(c_{2}-t\right)^{2} & \frac{1}{2} c_{2} \leq t \leq c_{2} \\ 0 & c_{2} \leq t\end{cases}
$$

Its maximum is attained for

$$
t=\frac{c_{2}}{3} \text { and is } \sigma_{T}(0)=\rho_{T}\left(0, c_{2} / 3\right)=\frac{1}{3} .
$$

It can be observed that by performing a shear, the general case $0 \leq c_{1} \leq 1$ can be reduced to the special case when $c_{1}=1$, which means that the line is orthogonal to the side $[a, b]$. In turn this is equivalent to the special case when $c_{1}=1$ in Proposition 5. Anyway, the proof is very simple even if we assume only that $0 \leq c_{1} \leq 1$, and is therefore omitted.

If one of the adjacent angles is larger than $\frac{1}{2} \pi$, the best result is smaller than $1 / 3$ :

Proposition 3 Let $T$ be the triangle with vertices at $a=(0,0), b=(1,0), c=$ $\left(c_{1}, c_{2}\right)$, assuming now $c_{1} \geq 1$ and $c_{2}>0$. Then the relative area of

$$
T_{Y(0, t)}=T \cap T^{[\partial Y(0, t)]} \cap Y(0, t),
$$

where the half plane $Y(0, t)$ is defined by $x_{2} \leq t$, is

$$
\rho_{T}(0, t)=\frac{\operatorname{area}\left(T_{Y(0, t)}\right)}{\operatorname{area}(T)}= \begin{cases}0 & t \leq 0 \\ \frac{1}{2 c_{1}+1}-\frac{2 c_{1}+1}{c_{2}^{2}}\left(t-\frac{c_{2}}{2 c_{1}+1}\right)^{2}, & 0 \leq t \leq \frac{c_{2}}{2 c_{1}} \\ \frac{1}{c_{2}^{2}\left(2 c_{1}-1\right)}\left(c_{2}-t\right)^{2} & \frac{c_{2}}{2 c_{1}} \leq t \leq c_{2} \\ 0 & c_{2} \leq t .\end{cases}
$$


Its maximum is attained for

$$
t=\frac{c_{2}}{2 c_{1}+1} \text { and is } \sigma_{T}(0)=\rho_{T}\left(0, c_{2} /\left(2 c_{1}+1\right)\right)=\frac{1}{2 c_{1}+1} .
$$

Thus, in both cases,

$$
\sigma_{T}(0)=\min \left(\frac{1}{3}, \frac{1}{2 c_{1}+1}\right), \quad c_{1} \geq \frac{1}{2} .
$$

The proof of this proposition is also easy and is omitted here.

When $c_{1} \nearrow+\infty$, equivalently $\beta \nearrow \pi$, the best folding becomes very bad: the relative area of the doubly covered set tends to 0

Corollary 1 If no angle in a triangle is larger than $\frac{1}{2} \pi$, then the best result for the area of the doubly covered object when folding along a line parallel to a side is $\frac{1}{3}$ in all three cases.

If one angle, say $\beta$, is larger than $\frac{1}{2} \pi$, then folding along a line parallel to $[a, b]$, $[b, c]$, and $[c, a]$ yields, respectively, the best result

$$
\frac{1}{2\|c-a\|_{2} \cos \alpha+1}<\frac{1}{3}, \frac{1}{2\|c-a\|_{2} \cos \gamma+1}<\frac{1}{3} \text {, and } \frac{1}{3} \text {. }
$$

\section{Folding along a line parallel to a bisector}

Proposition 4 Let $T$ be the triangle with vertices at $a=(0,0), b=\left(b_{1}, b_{2}\right), c=$ $\left(c_{1}, c_{2}\right)$, assuming $c_{1}<0<b_{1}$ and $0<c_{2} \leq b_{2}$. Assume that the line $L(-\pi / 2, t)$, $i$. e., the line of equation $x_{1}=t$, is parallel to the bisector between the sides $[a, b]$ and $[a, c]$. It follows that $c_{2} / c_{1}=-b_{2} / b_{1}$. Then the relative area of $T_{Y(-\pi / 2, t)}=$ $T \cap T^{[\partial Y(-\pi / 2, t)]} \cap Y(-\pi / 2, t)$ is

$$
\rho_{T}(-\pi / 2, t)=\frac{\operatorname{area}\left(T_{Y(-\pi / 2, t)}\right)}{\operatorname{area}(T)}= \begin{cases}0 & t \leq c_{1} \\ K_{1}\left(t-c_{1}\right)^{2}, & c_{1} \leq t \leq 0 \\ K_{2}\left(b_{1}-t\right)^{2} & 0 \leq t \leq b_{1} \\ 0 & b_{1} \leq t\end{cases}
$$

where

$$
K_{1}=\frac{b_{1}}{c_{1}^{2}\left(b_{1}-c_{1}\right)} \text { and } K_{2}=\frac{1}{b_{1}\left(b_{1}-c_{1}\right)} .
$$

Its maximum is attained for

$$
t=0 \text { and is } \sigma_{T}(-\pi / 2)=\rho_{T}(-\pi / 2,0)=\frac{-c_{1}}{b_{1}-c_{1}}=\frac{c_{2}}{b_{2}+c_{2}}=\frac{B}{B+C} .
$$


Proof. It is clear that, for $c_{1} \leq t \leq 0$, the value of the doubly covered area is proportional to the the square of $t-c_{1}$, and similarly proportional to $\left(b_{1}-t\right)^{2}$ for $0 \leq t \leq b_{1}$. To determine $K_{1}$ and $K_{2}$ we only have to calculate $\rho_{T}(-\pi / 2,0)=K_{1} c_{1}^{2}=K_{2} b_{1}^{2}$.

We see that the side $[a, b]$ reflected in the line $L(-\pi / 2, t)$ is parallel to the side $[a, c]$, and this accounts for the discontinuity in the derivative of $\rho_{T}(-\pi / 2, t)$ for $t=0$.

Corollary 2 If we fold a triangle with side lengths $A \leq B \leq C$ along the bisector of its smallest angle, then the relative area of the doubly covered set is $B /(B+C)$. Similarly, folding along the bisector of the angle opposite to $B$ yields $A /(A+C)$. If we fold along the bisector of the largest angle, the result is $A /(A+B)$. If the lengths of the sides of a triangle are $A, B$ and $C$ with $A \leq B \leq C$ and we fold along lines through a vertex, the maximum of $\operatorname{area}\left(T_{Y}\right) / \operatorname{area}(T)$ is

$$
\max \left(\frac{B}{B+C}, \frac{A}{A+B}\right)=\max \left(\frac{1}{1+\sin \gamma / \sin \beta}, \frac{1}{1+\sin \beta / \sin \alpha}\right) .
$$

It follows that

$$
\operatorname{BF}^{\text {vertex }}(T)=\max \left(\frac{1}{1+\sin \gamma / \sin \beta}, \frac{1}{1+\sin \beta / \sin \alpha}\right),
$$

and that the universal constant $\mathrm{WBF}^{\mathrm{vertex}}$ is equal to

$$
\mathrm{WBF}^{\mathrm{vertex}}=\frac{3}{2}-\frac{1}{2} \sqrt{5} \approx 0.3820
$$

Since the constant $\mathrm{WBF}^{\mathrm{vertex}}$ is $\frac{3}{2}-\frac{1}{2} \sqrt{5}$, the constant WBF is larger than or equal to this number.

Proof. There are three bisectors, and Proposition 4 shows that the best we can do is the maximum of the three quantities is $B /(B+C), A /(A+B)$ and $A /(A+C)$. However, the last quantity is always majorized by each of the other two.

The bad triangles for this kind of folding are $T_{\varepsilon}^{\mathrm{vertex}}=T(a, b, c)$ in standard coordinates with $c_{1}=\mathbf{A u}=\frac{1}{2} \sqrt{5}-\frac{1}{2} \approx 0.6180$ and $c_{2}=\varepsilon$.

We see that $\mathrm{BF}^{\text {vertex }}(T)=B /(B+C)$ when the middle side is large, meaning that $B \geq \sqrt{A C}$, the geometric mean of the lengths of the two other sides. To get a good result, we then fold along the bisector of the smallest angle, $\alpha$. When the middle side is small, meaning that $B \leq \sqrt{A C}$, then $\mathrm{BF}^{\text {vertex }}(T)=A /(A+B)$. Then we fold along the bisector of the largest angle, $\gamma$. In particular, when $A \approx 1-\frac{1}{2} \sqrt{2}$, $B \approx \frac{1}{2} \sqrt{2}$, and $C=1$, then $B>\sqrt{A C}$, so the best result is obtained by folding along the bisector at $a$, yielding $B /(B+C) \approx \sqrt{2}-1$. 
While the infimum of all $\mathrm{BF}^{\mathrm{vertex}}(T)$ is $\frac{1}{2}(3-\sqrt{5})$, we can see easily that equality cannot occur in the inequality $\operatorname{BF}^{\mathrm{vertex}}(T)>\frac{1}{2}(3-\sqrt{5})$, for if so, we must have

$$
\max \left(\frac{B}{B+C}, \frac{A}{A+B}\right)=\frac{1}{2}(3-\sqrt{5}),
$$

which implies that $C / B \geq \frac{1}{2}(\sqrt{5}+1), B / A \geq \frac{1}{2}(\sqrt{5}+1)$, which in turn implies that $A+B \leq C$, contradicting the strict triangle inequality $C<A+B$.

\section{Folding along a line orthogonal to a side}

Proposition 5 Let $T$ be a triangle in standard coordinates (8). We fold along a line $L(-\pi / 2, t)=L(\pi / 2,-t)$, i. e., of equation $x_{1}=t$, orthogonal to $[a, b]$. Then the relative area $\rho_{T}(-\pi / 2, t)$ of the triangle $T_{Y(\pi / 2,-t)}$ is

$$
\frac{\operatorname{area}\left(T_{Y(-\pi / 2, t)}\right)}{\operatorname{area}(T)}= \begin{cases}0 & t \leq 0 ; \\ \frac{1}{c_{1}} t^{2}, & 0 \leq t \leq \frac{1}{2} ; \\ \frac{1}{2 c_{1}+1}-\frac{2 c_{1}+1}{c_{1}\left(2 c_{1}-1\right)}\left(t-\frac{2 c_{1}}{2 c_{1}+1}\right)^{2}, & \frac{1}{2} \leq t \leq c_{1} ; \\ \frac{1}{1-c_{1}}(1-t)^{2}, & c_{1} \leq t \leq 1 ; \\ 0 & 1 \leq t .\end{cases}
$$

The function $t \longmapsto \rho_{T}(-\pi / 2, t)$ is a spline function of degree 2 . Its derivative at the nodes $t=0,1 / 2, c_{1}, 1$ has the values $0,1 / c_{1},-2$, and 0 , respectively. Its maximum is attained for

$t=\frac{2 c_{1}}{2 c_{1}+1}$ and is $\sigma_{T}(\pi / 2)=\sigma_{T}(-\pi / 2)=\rho_{T}\left(-\pi / 2,2 c_{1} /\left(2 c_{1}+1\right)\right)=\frac{1}{2 c_{1}+1}$.

By choosing $c_{1}<1$ close to 1 we can obtain that the best relative area is just a little bit larger than $\frac{1}{3}$, similarly to the result when we fold along a line parallel to a side; see Proposition 2. In fact, the bad triangles for this kind of folding are $T_{\varepsilon}^{\perp}=T(a, b, c)$ with $c_{1}=1-\varepsilon$ and $c_{2}=\varepsilon^{2}$. Then folding along a normal to $[a, b]$ yields $1 /\left(2 c_{1}+1\right)=1 /(3-2 \varepsilon) \searrow \frac{1}{3}$ as $\varepsilon \searrow 0$. Foldings along normals to $[b, c]$ or $[c, a]$ give worse results since $\gamma>\frac{1}{2} \pi$. Therefore $\mathrm{WBF}^{\perp}=\frac{1}{3}$.

If we choose $c_{1}=\frac{1}{2} \sqrt{2}$, the largest relative area is $\sqrt{2}-1$, attained for $t=t_{\max }=2-\sqrt{2}$. The expression for $t \in[1 / 2,1 / \sqrt{2}]$ is

$$
\rho_{T}(-\pi / 2, t)=\sqrt{2}-1-(4+3 \sqrt{2})\left(t-t_{\max }\right)^{2}, \quad \frac{1}{2} \leq t \leq \frac{1}{2} \sqrt{2} .
$$

We get the following values of $\rho_{T}(-\pi / 2, t)$ for three values of $t$ :

$$
\begin{array}{lll}
t=0.5 & \text { yields } & \rho_{T}(-\pi / 2, t)=\frac{1}{4} \sqrt{2} \approx 0.3536 ; \\
t=t_{\max }=2-\sqrt{2} \approx 0.5858 & \text { yields } & \rho_{T}(-\pi / 2, t)=\sigma_{T}(-\pi / 2)=\sqrt{2}-1 \\
& & \approx 0.4142 ; \\
t=c_{1}=\frac{1}{2} \sqrt{2} \approx 0.7071 & \text { yields } & \rho_{T}(-\pi / 2, t)=1-\frac{1}{2} \sqrt{2} \approx 0.2929 .
\end{array}
$$


Proof. The formulas for $t \leq \frac{1}{2}$ and $c_{1} \leq t$ are easily found.

For $\frac{1}{2}<t<c_{1}$, the two segments $\left[(2 t, 0),\left(t, c_{2} t / c_{1}\right)\right]$ and $\left[\left(c_{1}, c_{2}\right),(1,0)\right]$ intersect at a point $s=\left(s_{1}, s_{2}\right)$ with

$$
s_{1}=\frac{\frac{1}{2} c_{1}-\left(1-c_{1}\right) t}{c_{1}-\frac{1}{2}}, \quad s_{2}=c_{2} \frac{2 t-1}{c_{1}-\frac{1}{2}} .
$$

We shall calculate the area of the doubly covered figure, which is the quadrilateral $Q(p, r, s, q)$ with vertices at $p=(t, 0), r=b=(1,0), s=\left(s_{1}, s_{2}\right)$ just defined, and $q=\left(t, c_{2} t / c_{1}\right)$.

By (17), twice the area of $Q(p, r, s, q)$ is given by the formula

$$
\begin{aligned}
& \operatorname{det}\left(\begin{array}{ll}
t & 1 \\
0 & 0
\end{array}\right)+\operatorname{det}\left(\begin{array}{ll}
1 & s_{1} \\
0 & s_{2}
\end{array}\right)+\operatorname{det}\left(\begin{array}{cc}
s_{1} & t \\
s_{2} & c_{2} t / c_{1}
\end{array}\right)+\operatorname{det}\left(\begin{array}{cc}
t & t \\
c_{2} t / c_{1} & 0
\end{array}\right) \\
& =0+s_{2}+\frac{c_{2} s_{1} t}{c_{1}}-s_{2} t-\frac{c_{2} t^{2}}{c_{1}},
\end{aligned}
$$

which divided by $c_{2}$, twice the area of the triangle, gives the second-degree polynomial in the statement of the proposition. The derivative is zero at $t=2 c_{1} /\left(2 c_{1}+1\right)$ and this yields the maximum area.

When $c_{1}-1 / 2$ is a small positive number, the second derivative of $\rho_{T}$ with respect to $t$ in the interval $\left[1 / 2, c_{1}\right]$ is a large negative number. When $c_{1}=1 / 2$, we pass directly from the expression $2 t^{2}$ for $0 \leq t \leq 1 / 2$ to $2(1-t)^{2}$ for $1 / 2 \leq t \leq 1$; there is a jump in the first derivative.

We note that, when $c_{1}>1 / 2$, the maximum is not attained when the line passes through the vertex $\left(t=c_{1}\right)$ but at a point $t$ to the left of $c_{1}: 1 / 2<t=2 c_{1} /\left(2 c_{1}+\right.$ 1) $<c_{1}$.

If we want to find only the maximum of $\rho_{T}(-\pi / 2, t)$, we can observe that, using the notation in the proof, it is attained when $c_{2}\left(s_{1}-t\right) / c_{1}$ is half of $q_{2}=c_{2} t / c_{1}$.

\section{Combining two methods of folding}

We have seen that folding through a vertex and folding orthogonally against a side sometimes yield good results, but for different shapes. We now combine the two methods.

Theorem 2 Any triangle can be folded along a straight line such that the area of the doubly covered part is at least $\sqrt{2}-1 \approx 0.4142$. This bound can be obtained by using one of two methods: folding along the bisector of the smallest angle and folding orthogonally to the largest side. This proves the first part of the main result. 
In standard coordinates, the best relative area that can be obtained by these two methods is

$$
\Phi(c)=\max \left(\frac{1}{2 c_{1}+1}, \frac{1}{1+1 /\|c\|_{2}}\right), \quad c \in \Gamma .
$$

The values of $\Phi$ satisfy $\sqrt{2}-1<\Phi(c) \leq \frac{1}{2}$. The infimum of $\Phi$ is $\sqrt{2}-1$. It is not attained, but is the limit as $c$ approaches the boundary point $c=\left(\frac{1}{2} \sqrt{2}, 0\right) \in \partial \Gamma$.

Remark 1 We can see from the expression for $\Phi$ that folding along a line orthogonal to the longest side gives a strictly better result than folding along a bisector if and only if $c \in \Gamma, \frac{1}{2} \leq c_{1} \leq \frac{1}{2} \sqrt{2}$, and $2 c_{1}\|c\|_{2}<1$. They give the same result on a portion of the four-degree curve of equation $4 c_{1}^{2}\left(c_{1}^{2}+c_{2}^{2}\right)=1$. In polar coordinates $\left(c_{1}, c_{2}\right)=(r \cos \varphi, r \sin \varphi)$, the equation of the curve is $2 r^{2} \cos \varphi=1,0 \leq \varphi \leq \frac{1}{3} \pi$. In particular, if $B<\frac{1}{2} \sqrt{2}$, then $c_{1}<B<\frac{1}{2} \sqrt{2}$ and folding along a normal to the side $[a, b]$ gives $1 /\left(2 c_{1}+1\right)>\sqrt{2}-1$ while folding along the bisector at $a$ yields $B /(B+C)<\sqrt{2}-1$.

Proof. Let the notation be chosen so that $A \leq B \leq C$. We shall see that it is enough to fold along a line through $a$ or orthogonally against the side opposite to $c$.

Consider the two inequalities

$$
\begin{aligned}
& B \geq C / \sqrt{2} \\
& B^{2} \leq A^{2}+(\sqrt{2}-1) C^{2} .
\end{aligned}
$$

If we choose coordinates as in (8), these inequalities are equivalent to

$$
\begin{aligned}
& c_{1}^{2}+c_{2}^{2} \geq \frac{1}{2} \\
& c_{1} \leq \frac{1}{2} \sqrt{2}
\end{aligned}
$$

The first covers the permitted values of $c$ except those in the set with $c_{1}^{2}+c_{2}^{2}<\frac{1}{2}$ and $c_{2}>0$. These points are then taken care of by the second case.

If (21) holds, we fold along the bisector through $a$ and obtain from Corollary 2

$$
\mathrm{BF}^{\text {vertex }}(T)=\max \left(\frac{B}{B+C}, \frac{A}{A+B}\right) \geq \frac{B}{B+C} \geq \sqrt{2}-1 .
$$

If (21) does not hold, then (22) and (24) hold, and we can fold along a line orthogonal to the side $[a, b]$. We obtain $1 /\left(2 c_{1}+1\right) \geq \sqrt{2}-1$. 


\section{The constant found is best possible}

We shall now prove the second part of Theorem 1. This will be done in three steps.

First we shall prove that if the angle $\varphi$ of the line avoids the set $\frac{1}{2} \pi \mathbf{Z}$, then an easy estimate shows that the doubly covered set cannot have a relative area larger than $\sqrt{2}-1$ (Sect. 9.1). Next we shall study the behavior of the best folding when the angle is in a neighborhood of zero (Sect. 9.2); finally when the angle is in a neighborhood of $\frac{1}{2} \pi$ (Sect. 9.3).

The triangles which will provide us with examples are those which in standard coordinates have $c=\left(c_{1}, c_{2}\right)$ with $c_{1}=\frac{1}{2} \sqrt{2}$ and $c_{2}$ positive and very small. Equivalently, $c_{1}=\frac{1}{2} \sqrt{2}$ and the angle $\alpha$ is very small. The smaller $\varepsilon$ in the statement of Theorem 1 is, the smaller we have to take $\alpha$. This has the advantage that we can approximate to simplify many expressions, for example all of the quantities $c_{2} / c_{1}=\tan \alpha, \sin \alpha, \frac{1}{2} \sin 2 \alpha$ can be approximated by $\alpha+O\left(\alpha^{3}\right)$ as $\alpha \searrow 0$, which means that the error is not larger than a constant times $\alpha^{3}$ when $\alpha$ is positive and sufficently small; in fact for all $\alpha$ satisfying $0 \leq \alpha \leq \pi / 4$. Similarly, $\cos \alpha=1+O\left(\alpha^{2}\right)$ as $\alpha \searrow 0$. In the sequel we shall use these approximations many times, however without repeating the expression "as $\alpha \searrow 0$."

When $c_{1}=\frac{1}{2} \sqrt{2}$, we have

$$
\frac{B}{B+C}=\sqrt{2}-1+O\left(\alpha^{2}\right), \text { and } \frac{1}{2 c_{1}+1}=\sqrt{2}-1,
$$

implying that these two special methods of folding give approximately the same result when $\alpha$ is small. However, this is not sufficient: we now have to consider all kinds of folding.

\subsection{Folding along a line with angle avoiding $\frac{1}{2} \pi \mathrm{Z}$}

Proposition $7 \quad$ Let $T$ be a triangle in standard coordinates (8). With a half plane $Y(\varphi, t)$ and its boundary $L(\varphi, t)$ as defined in (3) and (4), assume that $\varphi \notin \frac{1}{2} \pi \mathbf{Z}$.

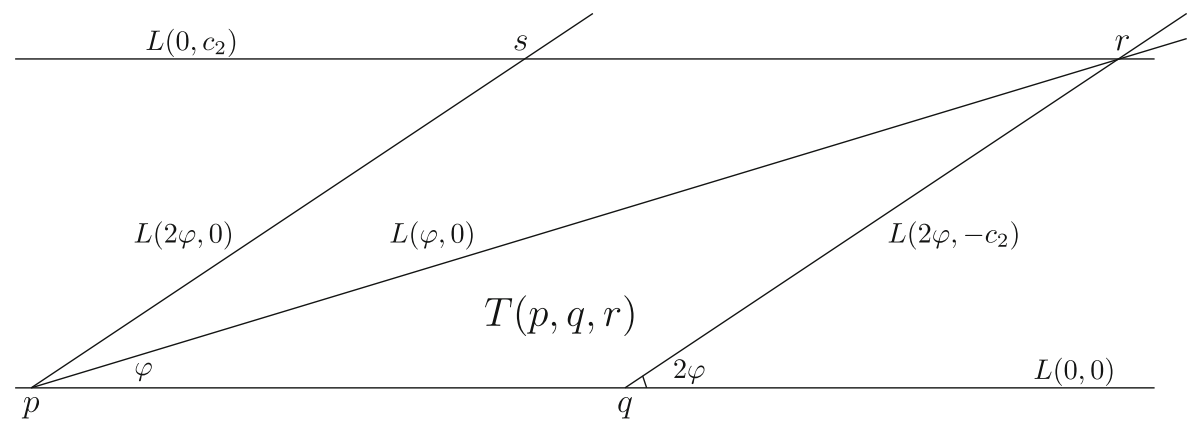

Fig. 2 We reflect the strip $S$ bounded by $L(0,0)$ and $L\left(0, c_{2}\right)$ in the line $L(\varphi, 0)$, here with $0<\varphi \leq$ $\pi / 4$, and put the reflected strip, which is bounded by $L(2 \varphi, 0)$ and $L\left(2 \varphi,-c_{2}\right)$, in the half plane $Y(\varphi, 0)$. The doubly covered area is the triangle $T(p, q, r)$. The original triangle (not shown) is $T(a, b, c)$ with $a=(0,0), b=(1,0)$ and $c_{2}>0$. In the rhomboid $Q(p, q, r, s)$ all sides are equally long; in particular $\|q-r\|_{2}=\|r-s\|_{2}=r_{1}-s_{1}$ 
Then the relative area of the doubly covered set when folding along $L(\varphi, t)$ is less than $c_{2} /|\sin 2 \varphi|$.

In the inequality for the relative area, we can either fix an angle $\varphi \notin \frac{1}{2} \pi \mathbf{Z}$ and choose a small $c_{2}$ to make the inequality $\operatorname{area}\left(T_{Y}\right) / \operatorname{area}(T)<c_{2} /|\sin 2 \varphi| \leq \sqrt{2}-1$ valid, or fix $c_{2}$ and consider the set of angles $\varphi$ which satisfy the inequality.

This means that when $|\sin 2 \varphi| \geq(\sqrt{2}+1) c_{2}$, the relative area is smaller than $\sqrt{2}-1$, implying that only a certain neighborhood of $\frac{1}{2} \pi \mathbf{Z}$ remains to be studied. This neighborhood is

$$
W\left(c_{2}\right)=\bigcup_{k \in \mathbf{Z}}\left\{\varphi \in \mathbf{R} ;\left|\varphi-\frac{1}{2} \pi k\right|<\eta\right\},
$$

where $\eta$, which depends on $c_{2}$, is given by the conditions $0<\eta<\frac{1}{4} \pi$ and $\sin 2 \eta=c_{2}(\sqrt{2}+1)$. When $\alpha$ is small, the angle $\eta$ is approximately equal to $\left(\frac{1}{2}+\frac{1}{4} \sqrt{2}\right) \alpha$; actually $\eta / \alpha$ tends to $\frac{1}{2}+\frac{1}{4} \sqrt{2} \approx 0.8535$ as $\alpha$ tends to zero.

Proof. We denote by $S$ the strip $S=\left\{x \in \mathbf{R}^{2} ; 0 \leq x_{2} \leq c_{2}\right\}$, bounded by $L(0,0)$ and $L\left(0, c_{2}\right)$. Since $T \subset S$, even strictly, the area of $T_{Y(\varphi, t)}$ is less than that of $S_{Y(\varphi, t)}$ (the area of $S^{Y(\varphi, t)}$ is infinite). Let now $\varphi \notin \frac{1}{2} \pi \mathbf{Z}$. It is enough to consider the case $0<\varphi \leq \frac{1}{4} \pi$; the other cases, like $-\frac{1}{2} \pi<\varphi \leq-\frac{1}{4} \pi,-\frac{1}{4} \pi \leq \varphi<0$, and $\frac{1}{4} \pi \leq \varphi<\frac{1}{2} \pi$ being similar. Then $S_{Y(\varphi, t)}$ is a triangle with vertices $p, q$ and $r$, given by

$p=\left(p_{1}, 0\right)$, the intersection of $L(0,0)$ and $L(\varphi, t)$;

$q=\left(q_{1}, 0\right)$, the intersection of $L(0,0)$ and the reflection of $L\left(0, c_{2}\right)$ in $L(\varphi, t)$;

$r=\left(r_{1}, c_{2}\right)$, the intersection of $L\left(0, c_{2}\right)$ and $L(\varphi, t)$.

We find that $p_{1}<q_{1}<r_{1}$ and that

$$
\sin 2 \varphi=\frac{c_{2}}{\|q-r\|_{2}}=\frac{c_{2}}{r_{1}-s_{1}}
$$

where $s=\left(s_{1}, c_{2}\right)$ is the reflection of $q$ in $L(\varphi, t)$. The area of $T_{Y(\varphi, t)}$ is therefore less than $\frac{1}{2} c_{2}\left(r_{1}-s_{1}\right)=\frac{1}{2} c_{2}^{2} / \sin 2 \varphi$. The area of $T$ is $\frac{1}{2} c_{2}$, so that the relative area is less than $c_{2} / \sin 2 \varphi$, a finite positive number.

\subsection{Folding with an angle near zero}

Consider a triangle $T(a, b, c)$ with standard coordinates (8) and intersect it with a line $L(\varphi, t)$. In view of Proposition 7 it is enough to study the lines $L(\varphi, t)$ with $\varphi$ either close to 0 or close to $\frac{1}{2} \pi$, more precisely, when $\varphi$ belongs to the intervals $[-\eta, \eta]$ and $[\pi / 2-\eta, \pi / 2+\eta]$, where $\eta$ is defined after formula (25). To do so, it is enough to use the approximations that follow. 
For $\varphi$ near 0 and in the neighborhood $W\left(c_{2}\right)$ defined by (25), we shall study angles $\varphi \in[-\eta, \eta]$. When $-\alpha \leq \varphi \leq 0$, we can use the results on folding along a line parallel to the bisector of the angle at $b$. Then the best result is

$$
\frac{A}{A+C} \approx \frac{3}{7}-\frac{1}{7} \sqrt{2} \approx 0.2265
$$

see Corollary 2. So this is much worse than the results for positive $\varphi$ to be discussed now. We study separately the cases $0 \leq \varphi \leq \frac{1}{2} \alpha$ and $\frac{1}{2} \alpha \leq \varphi \leq \alpha$.

Proposition 8 Let the angle $\varphi$ satisfy $0 \leq \varphi \leq \alpha$, and let $\varepsilon$ be any positive number. Then, for $\alpha$ small enough, the relative area of the doubly covered set $T_{Y}$ is never larger than $\sqrt{2}-1+\varepsilon$.

Proof. We reflect the line $L(\alpha, 0)$, which contains the side $[a, c]$, in the line $L(\varphi, t)$ to obtain the line $L(2 \varphi-\alpha, 2 t \cos (\varphi-\alpha))$. We reflect the line $L(-\beta, \sin \beta) \supset[c, b]$ in the line $L(\varphi, t)$ and obtain the line $L(2 \varphi+\beta, 2 t \cos (\varphi+\beta)-\sin \beta)$.

We then define four points $p, q, r, s$ by intersecting these lines:

$$
\begin{aligned}
& L(\varphi, t) \cap[a, c] \subset L(\varphi, t) \cap L(\alpha, 0)=\{p\} ; \\
& L(\varphi, t) \cap[c, b] \subset L(\varphi, t) \cap L(-\beta, \sin \beta)=\{q\} ; \\
& L(2 \varphi-\alpha, 2 t \cos (\varphi-\alpha)) \cap[a, b] \subset L(2 \varphi-\alpha, 2 t \cos (\varphi-\alpha)) \cap L(0,0)=\{r\} ; \\
& L(2 \varphi+\beta, 2 t \cos (\varphi+\beta)-\sin \beta) \cap[a, b] \subset \\
& \quad L(2 \varphi+\beta, 2 t \cos (\varphi+\beta)-\sin \beta) \cap L(0,0)=\{s\} .
\end{aligned}
$$

We have $c_{2}=c_{1} \tan \alpha=\left(1-c_{1}\right) \tan \beta$, so that

$$
\tan \beta=\frac{c_{1}}{1-c_{1}} \tan \alpha
$$

The triangles that are of interest for us now are the ones with $c_{1}=\frac{1}{2} \sqrt{2}$ and $c_{2}$ positive and very small. For these triangles we have good approximations,

$$
\begin{aligned}
c_{2} & =c_{1} \tan \alpha=\frac{1}{2} \sqrt{2} \alpha+O\left(\alpha^{3}\right) \approx 0.7071 \alpha, \\
\beta & =(1+\sqrt{2}) \alpha+O\left(\alpha^{3}\right) \approx 2.4142 \alpha, \\
\eta & =\left(\frac{1}{2}+\frac{1}{4} \sqrt{2}\right) \alpha+O\left(\alpha^{3}\right) \approx 0.8536 \alpha .
\end{aligned}
$$

For the line $L(\varphi, t)$ to actually intersect the sides $[a, c]$ and $[c, b]$, we must, for $0 \leq \varphi \leq \alpha$, have $t$ in the interval

$$
0 \leq t \leq-c_{1} \sin \varphi+c_{2} \cos \varphi=c_{1} \cos \varphi(\tan \alpha-\tan \varphi)=c_{1}(\alpha-\varphi)+O\left(\alpha^{3}\right),
$$

so also $t$ is of the order $\alpha$ when we study lines with angle $\varphi$ close to zero that actually cut the triangle.

The relative area, which is a polynomial in $t \in \mathbf{R}$ with coefficients that are rational functions of sines and cosines of $\alpha, \beta$ and $\varphi$, can therefore be approximated by a polynomial in $t$ with coefficients that are rational functions of $\alpha$ and $\varphi$. 
We obtain the following values for the coordinates of the points introduced above.

$$
\begin{aligned}
& p=\left(\frac{t \cos \alpha}{\sin (\alpha-\varphi)}, \frac{t \sin \alpha}{\sin (\alpha-\varphi)}\right)=\left(\frac{t}{\alpha-\varphi}, \frac{t \alpha}{\alpha-\varphi}\right)+O\left(\alpha^{2}\right) ; \\
& q=\left(\frac{\sin \beta \cos \varphi-t \cos \beta}{\sin (\varphi+\beta)}, \frac{\sin \beta \sin \varphi+t \sin \beta}{\sin (\varphi+\beta)}\right)=\left(\frac{\beta-t}{\beta+\varphi}, \frac{\beta \varphi+\beta t}{\beta+\varphi}\right)+O\left(\alpha^{2}\right) ; \\
& r=\left(r_{1}, 0\right)=\left(\frac{2 t \cos (\alpha-\varphi)}{\sin (\alpha-2 \varphi)}, 0\right)=\left(\frac{2 t}{\alpha-2 \varphi}, 0\right)+O\left(\alpha^{2}\right) ; \\
& s=\left(s_{1}, 0\right)=\left(\frac{\sin \beta-2 t \cos (\beta+\varphi)}{\sin (\beta+2 \varphi)}, 0\right)=\left(\frac{\beta-2 t}{\beta+2 \varphi}, 0\right)+O\left(\alpha^{2}\right) .
\end{aligned}
$$

Twice the area of $Q(p, r, s, q)$ is

$$
\begin{aligned}
& 2 \operatorname{area}(Q(p, r, s, q))=|p r|+|r s|+|s q|+|q p|=p_{2}\left(q_{1}-r_{1}\right)+q_{2}\left(s_{1}-p_{1}\right) \\
& =\frac{t \sin \alpha}{\sin (\alpha-\varphi)}\left[\frac{\sin \beta \cos \varphi-t \cos \beta}{\sin (\varphi+\beta)}-\frac{2 t \cos (\alpha-\varphi)}{\sin (\alpha-2 \varphi)}\right] \\
& \quad+\frac{\sin \beta \sin \varphi+t \sin \beta}{\sin (\varphi+\beta)}\left[\frac{\sin \beta-2 t \cos (\beta+\varphi)}{\sin (\beta+2 \varphi)}-\frac{t \cos \alpha}{\sin (\alpha-\varphi)}\right] .
\end{aligned}
$$

This is a polynomial in $t$ of order 2 , the coefficients of which are rational functions in the trigonometric functions of the angles indicated. Our task is to prove that this expression cannot exceed $2(\sqrt{2}-1) \operatorname{area}(T(a, b, c))=(\sqrt{2}-1) c_{2}$.

In the calculation to follow it is convenient to use the constant $K$,

$$
K=1+\sqrt{2} \approx 2.4142, \quad 1 / K=-1+\sqrt{2} \approx 0.4142, \quad K^{2}=3+2 \sqrt{2} \approx 5.8284
$$

and the variables

$$
\lambda=\frac{\varphi}{\alpha}, \quad 0 \leq \lambda \leq \frac{1}{2} ; \quad \tau=\frac{t}{\alpha}, \quad 0 \leq \tau \leq \frac{1}{2} \sqrt{2}(1-\lambda) \leq \frac{1}{2} \sqrt{2} .
$$

The points expressed in these constants and variables are:

$$
\begin{aligned}
& p=\left(\frac{\tau}{1-\lambda}, \frac{\alpha \tau}{1-\lambda}\right)+O\left(\alpha^{2}\right) ; \\
& q=\left(\frac{K-\tau}{K+\lambda}, \frac{K \alpha(\lambda+\tau)}{K+\lambda}\right)+O\left(\alpha^{2}\right) ; \\
& r=\left(r_{1}, 0\right)=\left(\frac{\tau}{\frac{1}{2}-\lambda}, 0\right)+O\left(\alpha^{2}\right) ; \\
& s=\left(s_{1}, 0\right)=\left(\frac{\frac{1}{2} K-\tau}{\frac{1}{2} K+\lambda}, 0\right)+O\left(\alpha^{2}\right) .
\end{aligned}
$$

Twice the area of the quadrilateral $Q(p, r, s, q)$ is

$$
\begin{gathered}
\operatorname{area}(Q(p, r, s, q))=|p r|+|r s|+|s q|+|q p|=p_{2}\left(q_{1}-r_{1}\right)+q_{2}\left(s_{1}-p_{1}\right) \\
=\frac{\alpha \tau}{1-\lambda}\left(\frac{K-\tau}{K+\lambda}-\frac{\tau}{\frac{1}{2}-\lambda}\right)+\frac{K \alpha(\lambda+\tau)}{K+\lambda}\left(\frac{\frac{1}{2} K-\tau}{\frac{1}{2} K+\lambda}-\frac{\tau}{1-\lambda}\right)+O\left(\alpha^{2}\right),
\end{gathered}
$$

while twice the area of the whole triangle is

$$
2 \operatorname{area}(T(a, b, c))=c_{2}=\frac{1}{2} \sqrt{2} \tan \alpha=\frac{1}{2} \sqrt{2} \alpha+O\left(\alpha^{3}\right),
$$


so that the relative area is

$$
\frac{2 \operatorname{area}(Q(p, r, s, q))}{2 \operatorname{area}(T(a, b, c))}=\rho_{T}(\lambda \alpha, \tau \alpha)=\frac{M_{0}+M_{1} \tau-M_{2} \tau^{2}}{D}+O(\alpha),
$$

where $D$ and the $M_{j}$ are given below. The zeros of $D$ are $1, \frac{1}{2}, v_{1}, v_{2}$, and $M_{2}$ is zero at $v_{3}$, where the $v_{j}$ are

$$
\begin{aligned}
& v_{1}=-K=-1-\sqrt{2} \approx-2.4142 ; \\
& v_{2}=-\frac{1}{2} K=-\frac{1}{2}-\frac{1}{2} \sqrt{2} \approx-1.2071 ; \\
& v_{3}=\frac{3}{2}+\frac{3}{4} \sqrt{2} \approx 2.5607 .
\end{aligned}
$$

We have

$$
\begin{aligned}
D & =(1-\lambda)\left(\frac{1}{2}-\lambda\right)\left(\lambda-v_{1}\right)\left(\lambda-v_{2}\right) ; \\
\text { and } & \\
M_{0} & =\frac{1}{2} K^{2} \sqrt{2} \lambda(1-\lambda)\left(\frac{1}{2}-\lambda\right)=\left(2+\frac{3}{2} \sqrt{2}\right) \lambda(1-\lambda)\left(\frac{1}{2}-\lambda\right) ; \\
M_{1} & =K^{2} \sqrt{2}(1-\lambda)\left(\frac{1}{2}-\lambda\right)=(4+3 \sqrt{2})(1-\lambda)\left(\frac{1}{2}-\lambda\right) ; \\
M_{2} & =(2+\sqrt{2})\left(\frac{3}{2}+\frac{3}{4} \sqrt{2}-\lambda\right)=(2+\sqrt{2})\left(v_{3}-\lambda\right) .
\end{aligned}
$$

So $D$ a polynomial in $\lambda$ of degree 4 , whereas the $M_{j}, j=0,1,2$, are polynomials in $\lambda$ of degree $3-j$. The signs are chosen so that $D$ and the $M_{j}$ are positive for $0<\lambda<\frac{1}{2}$.

Taking the supremum of the relative area over all $\tau$, we obtain

$$
\sigma_{T}(\varphi)=\sigma_{T}(\lambda \alpha)=\frac{M_{0}+\frac{M_{1}^{2}}{4 M_{2}}}{D}+O(\alpha)=\frac{4 M_{0} M_{2}+M_{1}^{2}}{4 D M_{2}}+O(\alpha) .
$$

Here the numerator is a polynomial in $\lambda$ of degree 4 and the denominator a polynomial of degree 5 .

However, the numerator here is divisible by $D$, so that the expression is equal to a constant divided by $M_{2}$, more precisely

$$
\sigma_{T}(\lambda \alpha)=\frac{\frac{1}{2}+\frac{1}{4} \sqrt{2}}{v_{3}-\lambda}+O(\alpha)=\frac{\frac{1}{2}+\frac{1}{4} \sqrt{2}}{\frac{3}{2}+\frac{3}{4} \sqrt{2}-\lambda}+O(\alpha)
$$

We write

$$
\sigma_{T}(\lambda \alpha)=S(\lambda)+O(\alpha)
$$

where

$$
S(\lambda)=\frac{\frac{1}{2}+\frac{1}{4} \sqrt{2}}{\frac{3}{2}+\frac{3}{4} \sqrt{2}-\lambda}, \quad \lambda \in[0,1 / 2],
$$


clearly an increasing function on $\left[0, \frac{1}{2}\right]$, which implies that on this interval, the supremum of $S$ is equal to the value $S\left(\frac{1}{2}\right)=\sqrt{2}-1$.

Since $\sigma_{T}(\lambda \alpha)$ and $S(\lambda)$ differ at most by a constant times $\alpha$, this result implies that, for any given positive number $\varepsilon$, the largest relative area of $T_{Y}$ is smaller than $\sqrt{2}-1+\varepsilon$ when $\alpha$ is sufficiently small.

A similar study can be made for $\frac{1}{2} \alpha \leq \varphi \leq \alpha$.

\subsection{Folding with an angle near $\pi / 2$}

We shall now study an angle $\varphi \in W\left(c_{2}\right)$ close to $\frac{1}{2} \pi$.

Proposition 9 Let us, in a triangle with standard coordinates (Sect. 4.2), fold along a line $L(\varphi,-t)$ with angle $\varphi$ satisfying $\left|\varphi-\frac{1}{2} \pi\right| \leq \alpha$, and let $\varepsilon$ be any positive number. Then, for $\alpha$ small enough, the relative area of the doubly covered set $T_{Y}$ is never larger than $\sqrt{2}-1+\varepsilon$.

Proof. It turns out that we can make a direct comparison of the folding along a line $L(\varphi,-t)$ with the folding along the line $L\left(\frac{1}{2} \pi,-q_{1}\right)=L\left(-\frac{1}{2} \pi, q_{1}\right)$, where $q=\left(q_{1}, q_{2}\right)$ is the point where $L(\varphi, t)$ intersects $[a, c]$. We already know that the relative area $\rho_{T}\left(-\frac{1}{2} \pi, q_{1}\right)$ is at most equal to $\rho_{T}\left(-\frac{1}{2} \pi, 2-\sqrt{2}\right)=\sqrt{2}-1$. The only values of $q_{1}$ that are of interest are those that satisfy $\frac{1}{2} \leq q_{1} \leq \frac{1}{2} \sqrt{2}$.

We introduce $\psi=\varphi-\frac{1}{2} \pi$, a small angle to which $q_{1}$ and $t$ are related by the equation

$$
-q_{1} \sin \varphi+q_{2} \cos \varphi=-q_{1} \cos \psi-q_{2} \sin \psi=-t .
$$

We reflect the line $L(0,0)$, which contains the side $[a, b]$, in the line $L(\varphi,-t)$ to obtain the line $L(2 \varphi,-2 t \cos \varphi)=L(2 \psi,-2 t \sin \psi)$.

We also reflect the line $L(\alpha, 0) \supset[c, a]$ in the line $L(\varphi,-t)$ and obtain the line

$$
L(2 \varphi-\alpha,-2 t \cos (\varphi-\alpha))=L(2 \psi-\alpha, 2 t \sin (\alpha-\psi)) .
$$

We then define four points $p, q, r, s$ by intersecting these lines:

$L(\varphi,-t) \cap[a, b] \subset L(\varphi,-t) \cap L(0,0)=\{p\} ;$

$L(\varphi,-t) \cap[c, a] \subset L(\varphi,-t) \cap L(\alpha, 0)=\{q\}$

$L(2 \psi,-2 t \sin \psi) \cap[b, c] \subset L(2 \psi,-2 t \sin \psi) \cap L(-\beta, \sin \beta)=\{r\} ;$

$L(2 \psi-\alpha, 2 t \sin (\alpha-\psi)) \cap[b, c] \subset L(2 \psi-\alpha, 2 t \sin (\alpha-\psi)) \cap L(-\beta, \sin \beta)=\{s\}$.

If $\psi<0$, then $r_{1}<0$ and $r$ is replaced by $b$; see Figs. 3 and 4 . 
The coordinates for these points are

$$
\begin{aligned}
& p=\left(\frac{t}{\cos \psi}, 0\right) ; \\
& q=\left(\frac{t \cos \alpha}{\cos (\alpha-\psi)}, \frac{t \sin \alpha}{\cos (\alpha-\psi)}\right) ; \\
& r=\left(\frac{\sin \beta \cos 2 \psi+2 t \sin \psi \cos \beta}{\sin (\beta+2 \psi)}, \frac{\sin \beta \sin 2 \psi-2 t \sin \psi \sin \beta}{\sin (\beta+2 \psi)}\right) ; \\
& s=\left(s_{1}, s_{2}\right), \text { where } \\
& s_{1}=\frac{\sin \beta \cos (2 \psi-\alpha)-2 t \sin (\alpha-\psi) \cos \beta}{\sin (\beta-\alpha+2 \psi)}, \text { and } \\
& s_{2}=\frac{\sin \beta \sin (2 \psi-\alpha)+2 t \sin (\alpha-\psi) \sin \beta}{\sin (\beta-\alpha+2 \psi)} .
\end{aligned}
$$

With $K=1+\sqrt{2}$ and $\lambda=\psi / \alpha$ the coordinates can be approximated as follows.

$$
\begin{aligned}
& p=(t, 0)+O\left(\alpha^{2}\right) \\
& q=(t, t \alpha)+O\left(\alpha^{2}\right) \\
& r=\left(\frac{\frac{1}{2} K+t \lambda}{\frac{1}{2} K+\lambda}, K \lambda \alpha \frac{1-t}{\frac{1}{2} K+\lambda}\right)+O\left(\alpha^{2}\right) \\
& s=\left(\frac{\frac{1}{2} K-t+t \lambda}{\frac{1}{2} \sqrt{2}+\lambda}, K \alpha \frac{\left.-\frac{1}{2}+t+\lambda-t \lambda\right)}{\frac{1}{2} \sqrt{2}+\lambda}\right)+O\left(\alpha^{2}\right) .
\end{aligned}
$$

\subsubsection{Angles less than $\pi / 2$}

For angles $\theta$ satisfying $0<\theta \leq \pi / 4$ we have

$$
0<\frac{2 \sqrt{2}}{\pi} \theta \leq \sin \theta<\theta<\tan \theta \leq \frac{4}{\pi} \theta .
$$

We shall apply these inequalities in some simple estimates. In particular we note that, with $c_{1}=\frac{1}{2} \sqrt{2}$, we must have $0<\alpha \leq \frac{1}{4} \pi$.

We first consider angles $\varphi<\frac{1}{2} \pi$ and compare the two quadrilaterals $Q(p, b, s, q)$ and $Q\left(p^{\perp}, b, s^{\perp}, q\right)$. Here $p^{\perp}=\left(q_{1}, 0\right)$, and $s^{\perp}$ is defined as the intersection of the side $[b, c]$ with the line obtained by reflecting the line $L(0,0)$ in the line $L\left(\frac{1}{2} \pi,-q_{1}\right)$.

The differences are (see Fig. 3):

$$
T\left(p, p^{\perp}, q\right)^{\circ} \subset Q(p, b, s, q) \backslash Q\left(p^{\perp}, b, s^{\perp}, q\right) \subset T\left(p, p^{\perp}, q\right)
$$

and

$$
T\left(q, s, s^{\perp}\right)^{\circ} \subset Q\left(p^{\perp}, b, s^{\perp}, q\right) \backslash Q(p, b, s, q) \subset T\left(q, s, s^{\perp}\right) .
$$

Since the interior of a triangle has the same area as the triangle itself, we see that

$$
\begin{aligned}
\operatorname{area}(Q(p, b, s, q))= & \operatorname{area}\left(Q\left(p^{\perp}, b, s^{\perp}, q\right)\right)+\operatorname{area}\left(T\left(p, p^{\perp}, q\right)\right) \\
& -\operatorname{area}\left(T\left(q, s, s^{\perp}\right)\right) .
\end{aligned}
$$




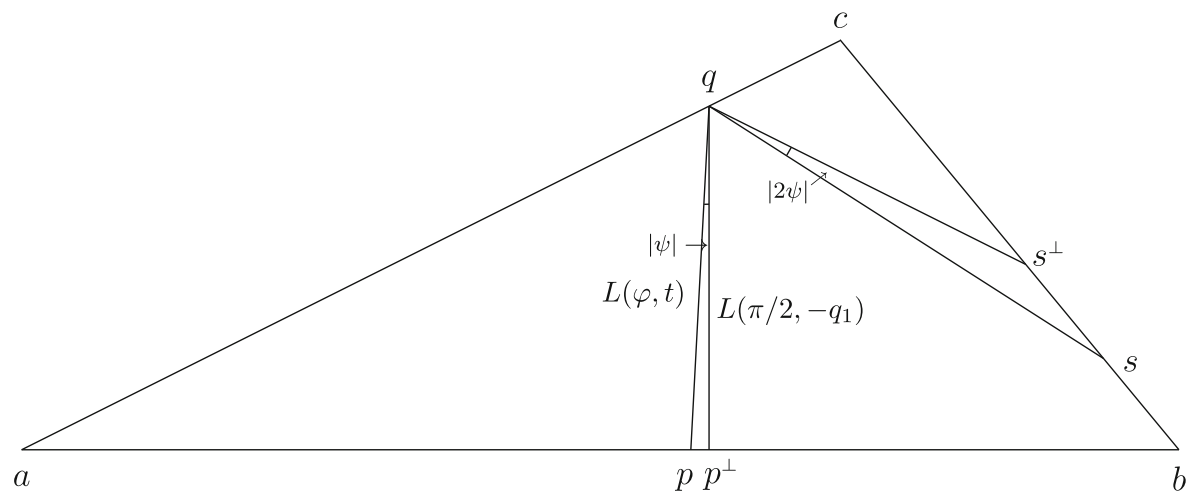

Fig. 3 Folding along a line $L(\varphi, t)$ with angle $\varphi=\frac{1}{2} \pi+\psi$ slightly smaller than a right angle and comparing it with folding along the line $L\left(\pi / 2,-q_{1}\right)$ with angle equal to $\pi / 2$ and intersecting the side $[c, a]$ in the same point $q$ as $L(\varphi, t)$. We compare $Q(p, b, s, q)$ with $Q\left(p^{\perp}, b, s^{\perp}, q\right)$

Here the second term is small: it is equal to

$$
\operatorname{area}\left(T\left(p, q^{\perp}, q\right)\right)=\frac{1}{2}\left(p_{1}^{\perp}-p_{1}\right) q_{2}=\frac{1}{2}|\tan \psi| q_{2}^{2} \leq \frac{2}{\pi}|\psi| q_{2}^{2},
$$

the angle at the vertex $q$ being $|\psi|$. Here

$$
q_{2}=q_{1} \tan \alpha \leq \frac{1}{2} \sqrt{2} \tan \alpha \leq \frac{2 \sqrt{2}}{\pi} \alpha .
$$

This implies that the area is at most

$$
\operatorname{area}\left(T\left(p, p^{\perp}, q\right)\right) \leq \frac{16}{\pi^{3}}|\psi| \alpha^{2} .
$$

The area of the whole triangle satisfies

$$
\frac{\sqrt{2}}{4} \alpha \leq \operatorname{area}(T(a, b, c))=\frac{1}{2} c_{2}=\frac{1}{2} c_{1} \tan \alpha \leq \frac{\sqrt{2}}{\pi} \alpha,
$$

implying that the relative area can be estimated from above as

$$
\frac{\operatorname{area}\left(T\left(p, p^{\perp}, q\right)\right)}{\operatorname{area}(T(a, b, c))} \leq \frac{32 \sqrt{2}}{\pi^{3}}|\psi| \alpha \leq \frac{3}{2}|\psi| \alpha=\frac{3}{2}|\lambda| \alpha^{2} .
$$

The other triangle, $T\left(q, s, s^{\perp}\right)$, has the area

$$
\operatorname{area}\left(T\left(q, s, s^{\perp}\right)\right)=\frac{1}{2}\|s-q\|_{2} \cdot\left\|s^{\perp}-q\right\|_{2} \cdot \sin 2|\psi| \geq \frac{2 \sqrt{2}}{\pi}\left(\frac{1}{2} \sqrt{2}-q_{1}\right)^{2}|\psi|,
$$

since 


$$
\|s-q\|_{2} \geq s_{1}-q_{1} \geq \frac{1}{2} \sqrt{2}-q_{1} \text { and also }\left\|s^{\perp}-q\right\|_{2} \geq s_{1}^{\perp}-q_{1} \geq \frac{1}{2} \sqrt{2}-q_{1} .
$$

This implies that the relative area is at least

$$
\left.\frac{\operatorname{area}\left(T\left(q, s, s^{\perp}\right)\right)}{\operatorname{area}(T(a, b, c))} \geq 2\left(\frac{1}{2} \sqrt{2}-q_{1}\right)^{2}\right)|\lambda| .
$$

We shall simplify equation (28) in two ways, depending on whether $q_{1}$ is closer to $2-\sqrt{2}$ or closer to $\frac{1}{2} \sqrt{2}$.

If $q_{1} \geq 1-\frac{1}{4} \sqrt{2}$ (the midpoint between of $2-\sqrt{2}$ and $\frac{1}{2} \sqrt{2}$ ), then $q_{1}-(2-\sqrt{2}) \geq$ $\frac{3}{4} \sqrt{2}-1$, and the relative area of $Q(p, b, s, q)$ is, in view of (20), at most

$$
\begin{aligned}
\sqrt{2} & -1-(4+3 \sqrt{2})\left[q_{1}-(2-\sqrt{2})\right]^{2}+\frac{3}{2}|\lambda| \alpha^{2} \\
& \leq \sqrt{2}-1-\left(\frac{3}{8} \sqrt{2}-\frac{1}{2}\right)+\frac{3}{2}|\lambda| \alpha^{2} \leq \sqrt{2}-1
\end{aligned}
$$

if

$$
\alpha^{2} \leq \frac{1}{4} \sqrt{2}-\frac{1}{3} \approx 0.02022
$$

If on the other hand, $q_{1} \leq 1-\frac{1}{4} \sqrt{2}$, then $\frac{1}{2} \sqrt{2}-q_{1} \geq \frac{3}{4} \sqrt{2}-1$, and the relative area of $Q(p, b, s, q)$ is, in view of (29) and (30), at most

$$
\sqrt{2}-1+|\lambda|\left[\frac{3}{2} \alpha^{2}-2\left(\frac{3}{4} \sqrt{2}-1\right)^{2}\right] \leq \sqrt{2}-1+|\lambda|\left(\frac{3}{2} \alpha^{2}-\frac{17}{4}+3 \sqrt{2}\right) \leq \sqrt{2}-1,
$$

if

$$
\alpha^{2} \leq \frac{17}{6}-2 \sqrt{2} \approx 0.0049
$$

We see that, in both cases, the relative area of $Q(p, b, s, q)$ is at most $\sqrt{2}-1$ for small values of $\alpha$.

\subsubsection{Angles larger than $\pi / 2$}

When $\psi=\varphi-\frac{1}{2} \pi>0$, we compare two quadrilaterals, the given one $Q(p, r, s, q)$ and the one obtained by decreasing the angle to $\frac{1}{2} \pi, Q\left(p^{\perp}, b, s^{\perp}, q\right)$, where as before $p^{\perp}=\left(q_{1}, 0\right)$, and $s^{\perp}$ is the intersection between the side $[b, c]$ and the line obtained as the reflection of the line $L(0,0)$ in the line $L\left(\frac{1}{2} \pi,-q_{1}\right)$.

The differences are

$$
T\left(q, s^{\perp}, s\right)^{\circ} \subset Q(p, r, s, q) \backslash Q\left(p^{\perp}, b, s^{\perp}, q\right) \subset T\left(q, s^{\perp}, s\right)
$$


and

$$
\begin{array}{r}
\left(T\left(p^{\perp}, p, q\right) \cup T(p, b, r)\right)^{\circ} \subset Q\left(p^{\perp}, b, s^{\perp}, q\right) \backslash Q(p, r, s, q) \\
\subset T\left(p^{\perp}, p, q\right) \cup T(p, b, r) ;
\end{array}
$$

see Fig. 4. We see that

$$
\begin{aligned}
& \operatorname{area}(Q(p, r, s, q)) \\
& \leq \operatorname{area}\left(Q\left(p^{\perp}, b, s^{\perp}, q\right)\right)+\operatorname{area}\left(T\left(q, s^{\perp}, s\right)\right)-\operatorname{area}\left(T(p, b, r)-\operatorname{area}\left(T\left(p^{\perp}, p, q\right)\right)\right. \\
& \leq \operatorname{area}\left(Q\left(p^{\perp}, b, s^{\perp}, q\right)\right)+\operatorname{area}\left(T\left(q, s^{\perp}, s\right)\right)-\operatorname{area}(T(p, b, r)) .
\end{aligned}
$$

We compare the second and the third terms. The second term is

$$
\operatorname{area}\left(T\left(q, s^{\perp}, s\right)\right)=\frac{1}{2}\left\|s^{\perp}-q\right\|_{2} \cdot\|s-q\|_{2} \cdot \sin 2 \psi,
$$

the angle at $q$ being $2 \psi$, and the third term is

$$
\operatorname{area}(T(p, b, r))=\frac{1}{2}\left(1-p_{1}\right)\|r-p\|_{2} \cdot \sin 2 \psi,
$$

the angle at $p$ also being $2 \psi$. We define

$$
V(\alpha, \psi, t)=\left(1-p_{1}\right)\|r-p\|_{2}-\left\|s^{\perp}-q\right\|_{2} \cdot\|s-q\|_{2} .
$$

Then the area of the quadrilateral $Q(p, r, s, q)$ can be estimated from above as

$$
\begin{aligned}
\operatorname{area}(Q(p, r, s, q)) & \leq \operatorname{area}\left(Q\left(p^{\perp}, b, s^{\perp}, q\right)\right)-\frac{1}{2} V(\alpha, \psi, t) \sin 2 \psi \\
& =\operatorname{area}\left(Q\left(p^{\perp}, b, s^{\perp}, q\right)\right)-\psi V(\alpha, \psi, t)+O\left(\alpha^{3}\right) .
\end{aligned}
$$

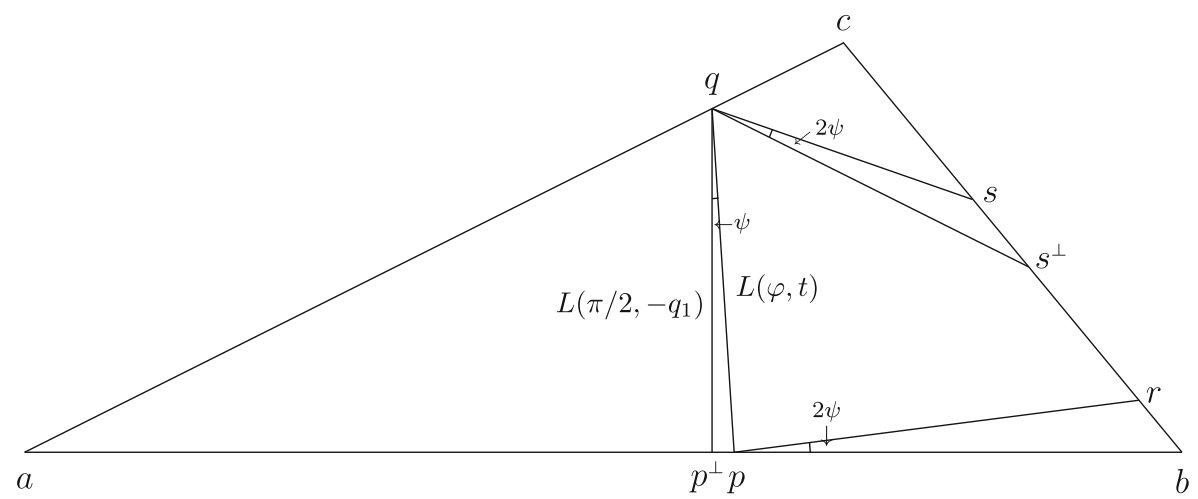

Fig. 4 Folding along a line $L(\varphi, t)$ with angle $\varphi=\frac{1}{2} \pi+\psi$ slightly larger than a right angle and comparing it with folding along the line $L\left(\pi / 2,-q_{1}\right)$ with angle equal to $\pi / 2$ and intersecting the side $[c, a]$ in the same point $q$ as $L(\varphi, t)$. We compare $Q(p, r, s, q)$ with $Q\left(p^{\perp}, b, s^{\perp}, q\right)$ 
We note that $s_{1}^{\perp}$ is an affine function of $q_{1}$ which takes the value 1 when $q_{1}=\frac{1}{2}$ and the value $\frac{1}{2} \sqrt{2}$ when $q_{1}=\frac{1}{2} \sqrt{2}$. It follows that

$$
s_{1}^{\perp}-q_{1}=1+\frac{1}{2} \sqrt{2}-(1+\sqrt{2}) q_{1}, \quad \frac{1}{2} \leq q_{1} \leq \frac{1}{2} \sqrt{2} .
$$

We compare the four quantities constituting $V$ with their limits as $\alpha$ tends to zero and indicate the deviation, which is at most a constant times some power of $\alpha$.

$$
\begin{aligned}
1-p_{1} & =1-q_{1}+O\left(\alpha^{2}\right)=1-t+O\left(\alpha^{2}\right) \\
\|r-p\|_{2} & =1-t+O(\alpha) \\
\left\|s^{\perp}-q\right\|_{2} & =s_{1}^{\perp}-q_{1}+O\left(\alpha^{2}\right)=1+\frac{1}{2} \sqrt{2}-(1+\sqrt{2}) q_{1}+O\left(\alpha^{2}\right) \\
\|s-q\|_{2} & =s_{1}^{\perp}-q_{1}+O(\alpha)=1+\frac{1}{2} \sqrt{2}-(1+\sqrt{2}) q_{1}+O(\alpha) .
\end{aligned}
$$

The limit of $V$ as $\alpha$ tends to zero is

$$
\lim _{\alpha \searrow 0} V(\alpha, \psi, t)=V(0,0, t)=(1-t)^{2}-\left[1+\frac{1}{2} \sqrt{2}-(1+\sqrt{2}) t\right]^{2},
$$

since also $\psi$ must tend to zero with $\alpha$, and in the limit $p_{1}$ and $q_{1}$ tend to $t$. The quantity $V(0,0, t)$ is nonnegative since we only consider $q_{1}$ in the interval $\frac{1}{2} \leq q_{1} \leq \frac{1}{2} \sqrt{2}$. With the estimates of the errors we have

$$
V(\alpha, \psi, t)=V(0,0, t)+O(\alpha) \geq O(\alpha)
$$

In view of (31) we can conclude that the area of $Q(p, r, s, q)$ is at most

$$
\operatorname{area}\left(Q\left(p^{\perp}, b, s^{\perp}, q\right)\right)-\psi V(0,0, t)+O\left(\alpha^{2}\right) \leq \operatorname{area}\left(Q\left(p^{\perp}, b, s^{\perp}, q\right)\right)+O\left(\alpha^{2}\right)
$$

implying that the relative area is at most $\sqrt{2}-1+O(\alpha) \leq \sqrt{2}-1+\varepsilon$ if $\alpha$ is small enough.

Open access funding provided by Uppsala University.

Open Access This article is distributed under the terms of the Creative Commons Attribution 4.0 International License (http://creativecommons.org/licenses/by/4.0/), which permits unrestricted use, distribution, and reproduction in any medium, provided you give appropriate credit to the original author(s) and the source, provide a link to the Creative Commons license, and indicate if changes were made.

\section{References}

1. Flachsmeyer, J.: Origami und Mathematik. Papier falten - Formen gestalten. Berliner Studienreihe zur Mathematik, vol. 20. Heldermann, Lemgo (2008). ISBN 978-3885381204. ii + 241 pp

2. Flachsmeyer, J.: Zu „Adolf Hurwitz faltet“. Math Semesterber 63(2), 195-200 (2016)

3. Haga, K.: Origamics. Mathematical explorations through paper folding. World Scientific Publishing, Hackensack (2008). xviii + 134 pp, edited and translated by Fonacier, J. C. and Isoda, M.

4. Jäger, J., Kroll, W., Schupp, H.: Blattfaltungen. Math Semesterber 61(1), 93-114 (2014)

5. Oswald, N.M.R.: Adolf Hurwitz faltet Papier. Math Semesterber 62(2), 123-130 (2015) 